\title{
Some Topics in Computational Algebraic Geometry
}

\author{
Frank-Olaf Schreyer
}

\begin{abstract}
Brief comments on selected topics in computational algebraic geometry are given. One of the topics is an experimental investigation of the possible Betti numbers of smooth canonical curves of low genus.

Key Words and Phrases: Computer algebra, Gröbner bases, syzygies, resolution of singularities, monodromy, Brieskorn lattice, Tate resolution, cohomology of coherent sheaves, Beilinson monads, invariants rings, binary forms, Green's conjecture, construction of canonical curves.
\end{abstract}

\section{Introduction}

Modern computer algebra systems allow to treat impressive examples in computational algebraic geometry. The basic mathematical tool are Gröbner bases as invented by Gordon (1899), Buchberger (1965), Hironaka (1964) and Grauert (1972). In particular Buchberger's algorithm to compute Gröbner bases is essential. For localization of polynomial rings this algorithm was adapted by Mora (1982). A rough classification of the applications is as follows:

(1) Elementary applications: ideal membership, normal forms, Hilbert function, dimension, degree, elimination, projective closure, tangent cone, syzygies, intersections, (I:J), $\operatorname{Hom}(\mathrm{M}, \mathrm{N})$.

(2) Modifications of algebraic sets: primary decomposition, normalization, Puiseux expansion, rational parameterization of curves (and surfaces), resolution of singularities.

(3) Homological methods: Ext, Tor, cohomology of coherent sheaves, Tate resolutions, monads, resultants.

(4) Parameter spaces: invariant rings, versal deformations of singularities and modules, special families: existence, uni-rationality.

(5) Enumerative geometry. 
(6) D-modules and topology: Bernstein-Sato polynomials, monodromy and Brieskorn lattices of isolated singularities, de Rham cohomo$\log y$.

In this survey we focus on few topics: resolution of singularities, monodromy, Tate resolutions, invariant theory and constructions of special families. For a more complete survey including a short treatment of basic Gröbner basis theory we refer to our survey Decker, Schreyer (2001).

\section{Resolution of singularities}

Gabor Bodnar and Josef Schicho (2000) implemented Villamayor's algorithm of resolution of singularities (see Encinas, Villamajor 1998) as part of a CASA package for Maple, mainly for surfaces and threefolds, see http://www.risc.uni-linz.ac.at. At the current state the algorithm is implemented for characteristic zero, but future implementation will include characteristic $p>0$, with the expectation, that the algorithm will work in many but not all cases. Note that the running time in characteristic $p>0$ might be shorter than for characteristic zero due to the fact that the coefficients in a Gröbner basis computation in characteristic $p>0$ do not accumulate.

The input are the polynomial equations of an affine scheme $Z$ embedded into a nonsingular affine subvariety $X$ of $\mathbf{A}^{n}$. The output is a tree of charts of blow-ups, whose final leaves consist of a covering of an embedded resolution $f: Y \rightarrow X$ of $Z$, all put together in an HTML document.

The number of charts, which are used to cover $Y$ even in simple examples can be large. For example the desingularisation of the Whitney umbrella $Z=\left\{z^{2}-x y^{2}=0\right\} \subset X=\mathbf{A}^{3}$ gives a tree with 50 nodes and 16 final leaves covering $Y$.

\section{Monodromy and Brieskorn lattices}

A SINGULAR package to compute the monodromy of an isolated hypersurface singularity has been developed by Mathias Schulze. It uses an algorithm by Brieskorn (1970) to compute a connection matrix of the meromorphic Gauss-Manin connection up to arbitrarily high order, and an algorithm of Gerard and Levelt (1973) to transform it to a simple pole.

The computation of the monodromy of the $D_{4}$ surface singularity in SINGULAR looks as follows:

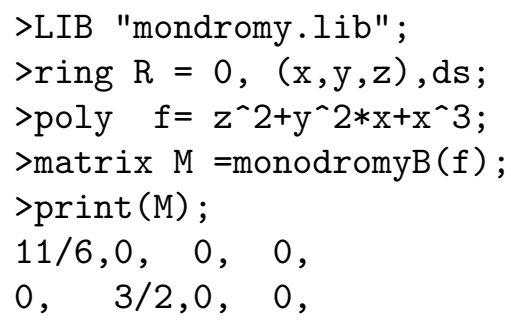


$0, \quad 0,3 / 2,0$

$0, \quad 0, \quad 0, \quad 7 / 6$

The monodromy operator is then $\exp (-2 \pi i M)$ in terms of the output matrix $M$.

\section{Tate resolution}

Bernstein, Gel'fand, Gel'fand (1978) established an equivalence between the derived category of coherent sheaves on $\mathbb{P}(W)$ and the stable module category of finitely generated graded modules over the graded exterior algebra $E=\Lambda V$, where $V=W^{*}$ are dual vector spaces over the ground field $K$. The heart of the construction associates to a graded $S=\operatorname{Sym}(W)$ module $M=\sum_{d} M_{d}$ the infinite linear complex

$$
\mathbf{R}(M): \quad \ldots \rightarrow \operatorname{Hom}_{K}\left(E, M_{d}\right) \rightarrow \operatorname{Hom}_{K}\left(E, M_{d+1}\right) \rightarrow \ldots
$$

and vice versa. In Eisenbud, Fløystad, Schreyer (2001) we review this construction starting from $\mathbf{R}(M)$. We obtain novel methods to compute cohomology of sheaves and to compute the Beilinson monad of a sheaf explicitely.

$\mathbf{R}\left(M_{\geq r}\right)$ becomes exact precisely for $r>\operatorname{reg}(M)$. Thus adjoining a free resolution of $\operatorname{ker}\left(R^{r}(M) \rightarrow R^{r+1}(M)\right)$, we may extend $\mathbf{R}\left(M_{\geq r}\right)$ to a doubly infinite exact complex of graded free $E$-modules

$$
\mathbf{T}(\tilde{M}) \quad \rightarrow T^{e} \rightarrow \ldots \rightarrow T^{r-1} \rightarrow T^{r}=R^{r}(M) \rightarrow T^{r+1}=R^{r+1}(M) \rightarrow \ldots
$$

which depends only on the sheaf $\mathcal{F}=\tilde{M}$.

Theorem 4.1 (Eisenbud, Fløystad, Schreyer 2001). For a coherent sheaf $\mathcal{F}=\tilde{M}$ on $\mathbb{P}(W)=\mathbb{P}^{n}$ we have

$$
T^{e}(\mathcal{F})=\sum_{i=0}^{n} \operatorname{Hom}_{K}\left(E, H^{i} \mathcal{F}(e-i)\right)
$$

where we regard $\mathrm{H}^{i} \mathcal{F}(e-i)$ as a vector space in degree $e-i$.

Thus syzygies over the exterior algebra allow to compute cohomology groups: Starting from the multiplication map

$$
M_{d} \otimes W \rightarrow M_{d+1}
$$

for sufficiently high $d$, we obtain one of the differentials of $\mathbf{R}(M)$ and a Gröbner basis calculation over the exterior algebra gives us any desired finite piece of $\mathbf{T}(\mathcal{F})$.

If we compare this with previous methods to compute cohomology, e.g.

$$
\mathrm{H}_{*}^{i}(\mathcal{F}) \cong \operatorname{Ext}_{S}^{n-i}\left(\Gamma_{*}(\mathcal{F}), S(-n-1)\right)^{*},
$$

then we see that to compute e.g. $\mathrm{H}^{1}$ we do not have to compute the complete free resolution of $\Gamma_{*}(\mathcal{F})$ but only some steps in the Tate resolution, which seems to be of more appropriate complexity. 
The differentials of $\mathbf{T}(\mathcal{F})$ are related to the Beilinson monad of $\mathcal{F}$, c.f. Beilinson (1978). Let $\Omega$ be the additive functor, which maps the free $E$ module $\omega_{E}(i)=\operatorname{Hom}_{K}(E, K(i))$ to the sheaf $\Omega^{i}(i)$, the twisted regular $i$ forms, and which maps morphisms via the identification

$$
\operatorname{Hom}_{E}\left(\omega_{E}(i), \omega_{E}(j)\right) \cong \Lambda^{i-j} V \cong \operatorname{Hom}_{\mathbb{P}(W)}\left(\Omega^{i}(i), \Omega^{j}(j)\right) .
$$

Then

Theorem 4.2 (Eisenbud, Fløystad, Schreyer 2001). $\Omega(\mathbf{T}(\mathcal{F})$ ) is the Beilinson monad for $\mathcal{F}$.

Thus the differentials of $\mathbf{T}(\mathcal{F})$ give us the differentials of the Beilinson monad. The differential of Beilinson monad were previously very difficult to compute explicitly.

Example. Consider the $2 \times 5$ matrix

$$
\varphi=\left(\begin{array}{lllll}
e_{1} e_{4} & e_{2} e_{0} & e_{3} e_{1} & e_{4} e_{2} & e_{0} e_{3} \\
e_{2} e_{3} & e_{3} e_{4} & e_{4} e_{0} & e_{0} e_{1} & e_{1} e_{2}
\end{array}\right)
$$

over the exterior algebra with generators $e_{0}, \ldots, e_{4}$. By direct computation we find the following Betti numbers in the Tate resolution of $\varphi$, where $\varphi$ sits in the indicated spot.

$$
\begin{array}{ccccccccccc}
100 & 35 & 4 & . & . & . & . & . & . & . & . \\
. & 2 & 10 & 10 & \mathbf{5} & . & . & . & . & . & . \\
. & . & . & . & . & \mathbf{2} & . & . & . & . & . \\
. & . & . & . & . & . & 5 & 10 & 10 & 2 & . \\
. & . & . & . & . & . & . & . & 4 & 35 & 100
\end{array}
$$

The Beilinson functor $\Omega$ picks out a finite complex

$$
0 \rightarrow \oplus^{5} \Omega^{4}(4) \rightarrow \oplus^{2} \Omega^{2}(2) \rightarrow \oplus^{5} \mathcal{O}_{\mathbb{P}^{4}} \rightarrow 0
$$

Its homology is the famous Horrocks-Mumford bundle (1973). It is easy to see from these Betti numbers, that it is the Tate resolution of a vector bundle, see Eisenbud, Fløystad, Schreyer (2001) for details.

\section{Invariant theory}

Let $G$ be a group and $\rho: G \rightarrow G L(V)$ a linear representation. The basic problem of invariant theory is to compute for $R=k[V]$ the $\operatorname{ring} R^{G}$ of invariant functions. If $G$ is reductive, then $R^{G}$ is a finitely generated $k$-algebra as proved by Hilbert in his first landmark paper (1890). Hilbert himself provided an algorithm to compute generators in his second landmark paper (1893), in which he introduced Noether normalization, the Hilbert-Mumford criterion and the Nullstellensatz, see also Sturmfels (1993) and Decker, de Jong (1999). A variant of Hilbert's original proof of finite generation was turned into an algorithm recently by Derksen (1999)

For finite groups this gives a reasonable good algorithm implemented by Decker and his group into SINGULAR. However for algebraic groups none of 
the algorithms works in practice so far, the reason being that at some step a too expensive elimination computation is required.

A computer implementation of Gordon's method for binary forms including covariants was done by Holger Cröni (2002). It can treat in reasonable time the case of binary septics, the case in which Sylvester's enumerative method predicted too few generators (1878). Later von Gall computed a complete system of invariants for binary septics in 1888. However von Gall got too many, which was finally corrected by Dixmier and Lazard (1986).

The weakest spot of Cröni's program is that the theoretical bounds for the degree of the generators are too large.

Clearly one would hope that the computation of invariant rings with Computer algebra improves upon the state of art a hundred years ago substantially. I think it is time to reconsider this problem from an algorithmic point of view.

\section{Constructions}

In this last section I would like to comment on computer algebra methods for constructions. For example one might want to prove, that a certain component of the Hilbert scheme is non-empty, and that its general points correspond to smooth varieties, or that the component is uni-rational. Computer algebra for this purpose was very successfully applied by Decker, myself and our students to the study of smooth non-general type surfaces in $\mathbb{P}^{4}$.

In this survey I will illustrate this method with an investigation of the possible Betti numbers for canonical curves.

Let $C \longrightarrow \mathbb{P}^{g-1}$ be the canonical morphism of a smooth curve of genus g. The syzygies of the canonical ring $R_{C}=\sum_{n \geq 0} H^{0}\left(C, \omega^{\otimes n}\right)$ as $S=$ $\operatorname{Sym}\left(H^{0}(C, \omega)\right)$ module are conjectured to be closely related to the BrillNoether theory of $C$. Since $R_{C}$ is Gorenstein, it has a self-dual resolution of length $g-2$. Moreover $R_{C}$ is 3 -regular. We summarize the numbers of generators of the modules $F_{i}=\sum_{j} S(-j)^{\beta_{i j}}$ in a minimal free resolution

$$
0 \leftarrow R_{C} \leftarrow F_{0} \leftarrow F_{1} \leftarrow \ldots \leftarrow F_{g-2} \leftarrow 0
$$

\section{in a Betti table}

$$
\begin{array}{cccccccccc}
1 & \cdot & \ldots & . & . & \ldots & . & \ldots & . & . \\
. & \beta_{12} & \ldots & * & \beta_{p+1, p+2} & \ldots & \beta_{g-2-p, g-1-p} & \ldots & & \beta_{02} \\
\beta_{02} & \beta_{13} & \ldots & \beta_{p, p+2} & * & \ldots & * & \ldots & \beta_{g-3, g-1} & \cdot
\end{array}
$$

Gorenstein gives the symmetry $\beta_{i j}=\beta_{g-2-i, g+1-j}$. The difference $\beta_{i+1, i+2}-$ $\beta_{i, i+2}$ depends only on $g$ and $i$ but not on the curve. The famous conjecture of Green gives a geometric interpretation of the range of nonzero $\beta_{i j}$ 's.

ConjeCture 6.1 (Green, 1984). Let $C$ be a smooth curve defined over C. Then $\beta_{p, p+2} \neq 0$ iff $C$ has Clifford index $\operatorname{Cliff}(C) \leq p$. 
The Clifford index of a line bundle $L$ is defined as

$$
\operatorname{Cliff}(L)=\operatorname{deg} L-2\left(h^{0}(L)-1\right)
$$

and the Clifford index of $C$ is

$$
\operatorname{Cliff}(C)=\min \left\{\operatorname{Cliff}(L) \mid L \text { a line bundle on } C \text { with } h^{0}(L), h^{1}(L) \geq 2\right\} .
$$

The direction from the existence of special line bundles to the existence of exceptional syzygies was established by Green and Lazarsfeld (1984). At present the conjecture is known for curves of genus $g \leq 9$, Mukai (1995). The case $p \leq 2$ is known by M. Noether (1880), Petri (1923), Voisin (1988), Schreyer (1991). Recently Voisin (2001) proved the conjecture for a general k-gonal curves of arbitrary genus except for the case of general curves of odd genus.

Thus it might be time to try to formulate a more precise version of the conjecture, i.e. to answer the question, which Betti tables actually occur for smooth curves. Also the conjecture is known to be false for fields of some finite characteristics. It is interesting to try to explain the exceptional characteristics for various genera. A computational approach to these questions runs along the following lines: Pick curves over finite fields at random, and compute their syzygies. For genus $g \leq 14$ it is possible to pick curves at random, as was shown in Schreyer, Tonoli (2001). Below I summarize, what I think are the possible Betti numbers for small $g$, and which are the exceptional characteristics. For $g \leq 8$ this was established in Schreyer (1986). For genus 9 we have:

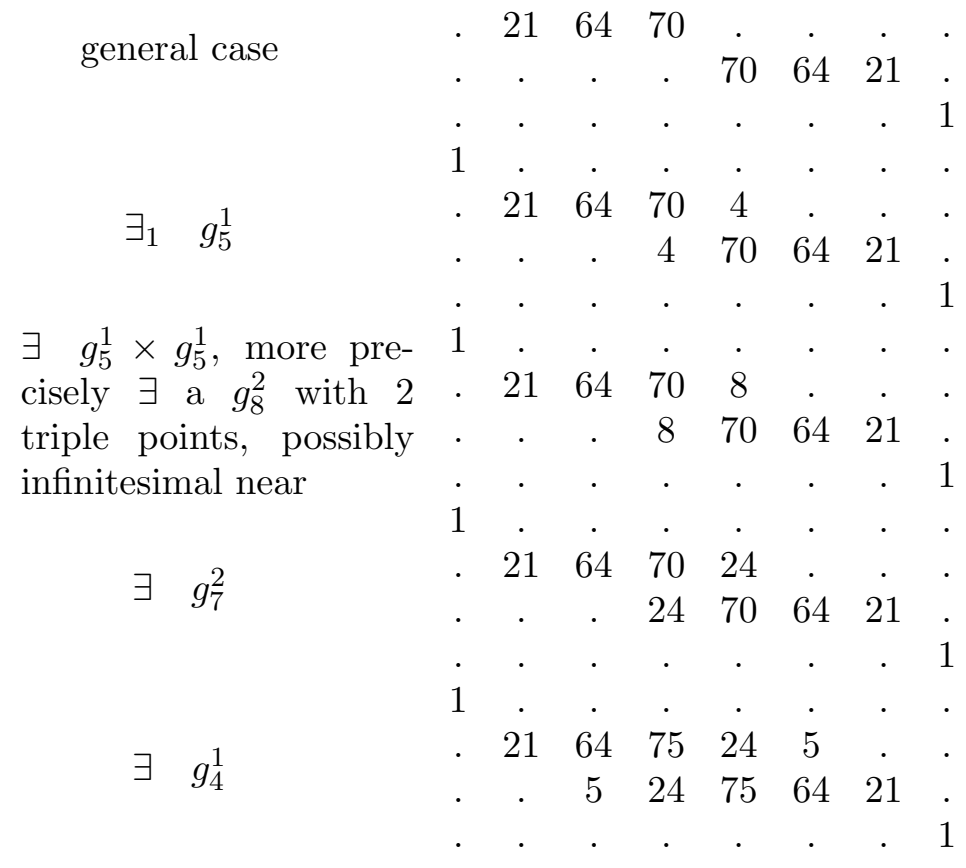




$$
\begin{aligned}
& \begin{array}{ccccccccc}
\exists g_{4}^{1} \times g_{5}^{1} & 1 & . & . & . & . & . & . & . \\
& . & 21 & 64 & 75 & 48 & 5 & . & . \\
& . & . & 5 & 48 & 75 & 64 & 21 & . \\
& . & . & . & . & . & . & . & 1
\end{array}
\end{aligned}
$$

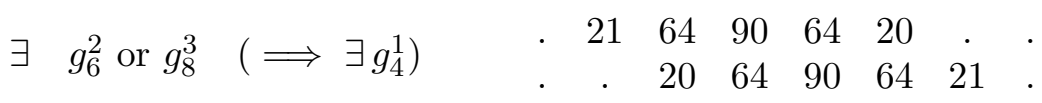

$$
\begin{aligned}
& \text {. . . . . . } 1 \\
& 1 \\
& \exists g_{3}^{1} \quad . \quad \begin{array}{ccccccc}
\exists & 70 & 105 & 84 & 35 & 6 & .
\end{array} . \\
& 1 . \quad . \quad . \quad . \quad .
\end{aligned}
$$

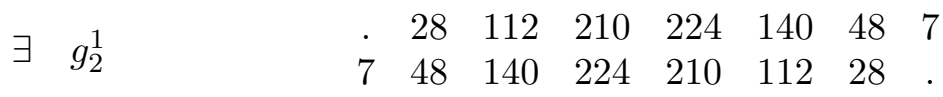

Table: Conjectural Betti numbers for genus 9 , characteristic $\neq 3$

It is not known whether this is the correct table for curves of Clifford index 3. For example the table claims that the existence of three $g_{5}^{1}$ 's implies the existence of a $g_{7}^{2}$.

In characteristic 3 the conjecture fails for the general curve. The following Betti numbers are possible for curves of genus 9 and Clifford index $\geq 3$ :

$$
\begin{array}{ccccccccc} 
& 1 & . & . & . & . & . & . & . \\
\text { general case } & . & 21 & 64 & 70 & 6 & . & . & . \\
& . & . & . & 6 & 70 & 64 & 21 & . \\
& . & . & . & . & . & . & . & 1 \\
& 1 & . & . & . & . & . & . & . \\
& . & 21 & 64 & 70 & 8 & . & . & . \\
& . & . & . & 8 & 70 & 64 & 21 & . \\
& . & . & . & . & . & . & . & 1 \\
& 1 & . & . & . & . & . & . & . \\
& . & 21 & 64 & 70 & 10 & . & . & . \\
& . & . & . & 10 & 70 & 64 & 21 & . \\
& . & . & . & . & . & . & . & 1 \\
& 1 & . & . & . & . & . & . & . \\
& . & 21 & 64 & 70 & 24 & . & . & . \\
& . & . & . & 24 & 70 & 64 & 21 & . \\
& . & . & . & . & . & . & . & 1
\end{array}
$$

Table: Conjectural Betti numbers for genus 9 in characteristic 3 
For genus 10 over a field of characteristic $\neq 3$ we find the following:

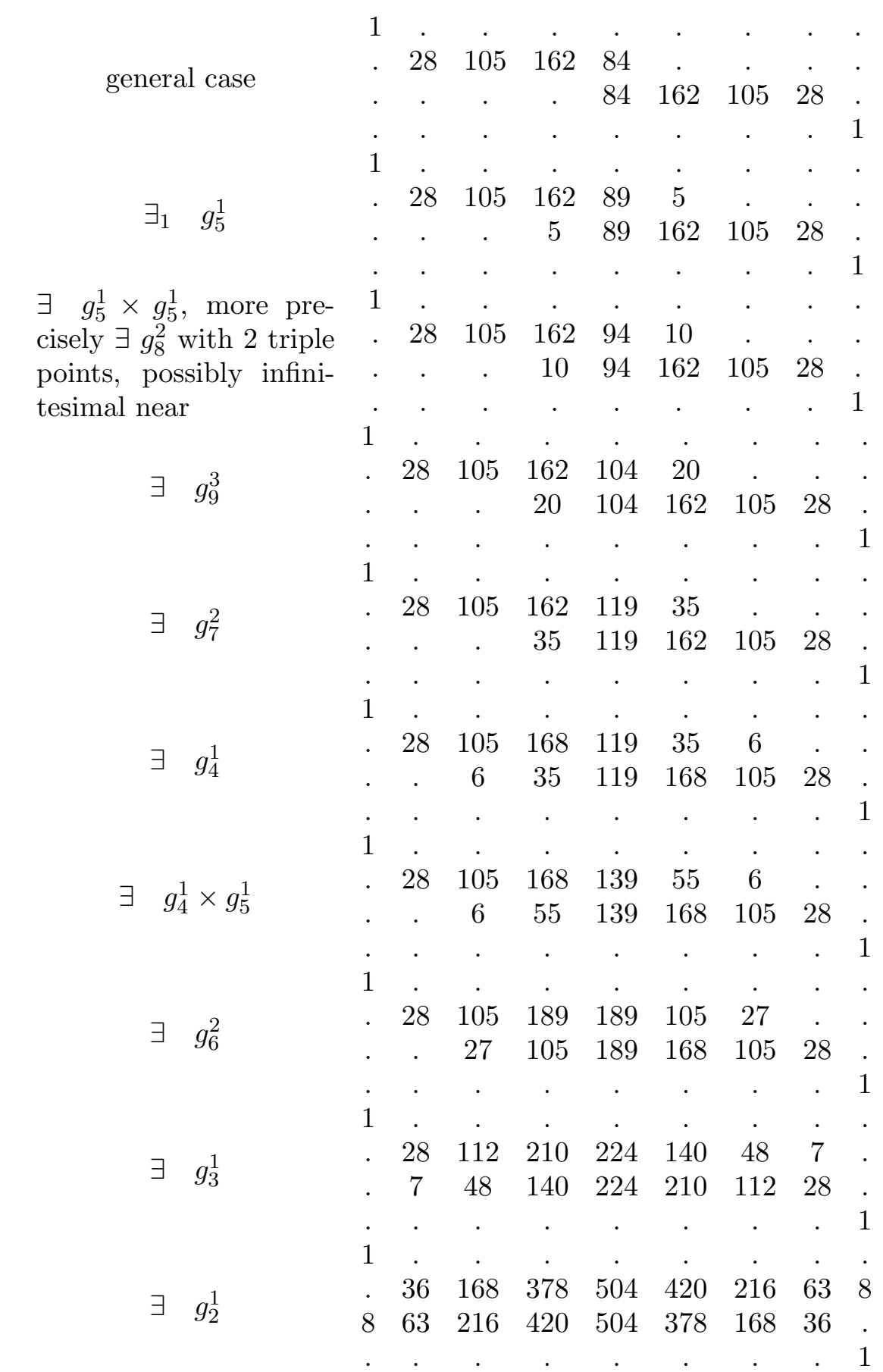

Table: Conjectural Betti numbers for genus 10 in characteristic $\neq 3$ 
The general curve of genus 10 over a field of characteristic 3 does not satisfy Green's conjecture.

$\begin{array}{lccccccccc} & 1 & . & . & . & . & . & . & . & . \\ \text { general case, } & . & 28 & 105 & 162 & 85 & 1 & . & . & . \\ \text { characteristic } 3 & . & . & . & 1 & 85 & 162 & 105 & 28 & . \\ & . & . & . & . & . & . & . & . & 1\end{array}$

In the case of genus 11 Green's conjecture does not hold in characteristic 2 and 3. For other characteristics the following Betti numbers are possible:

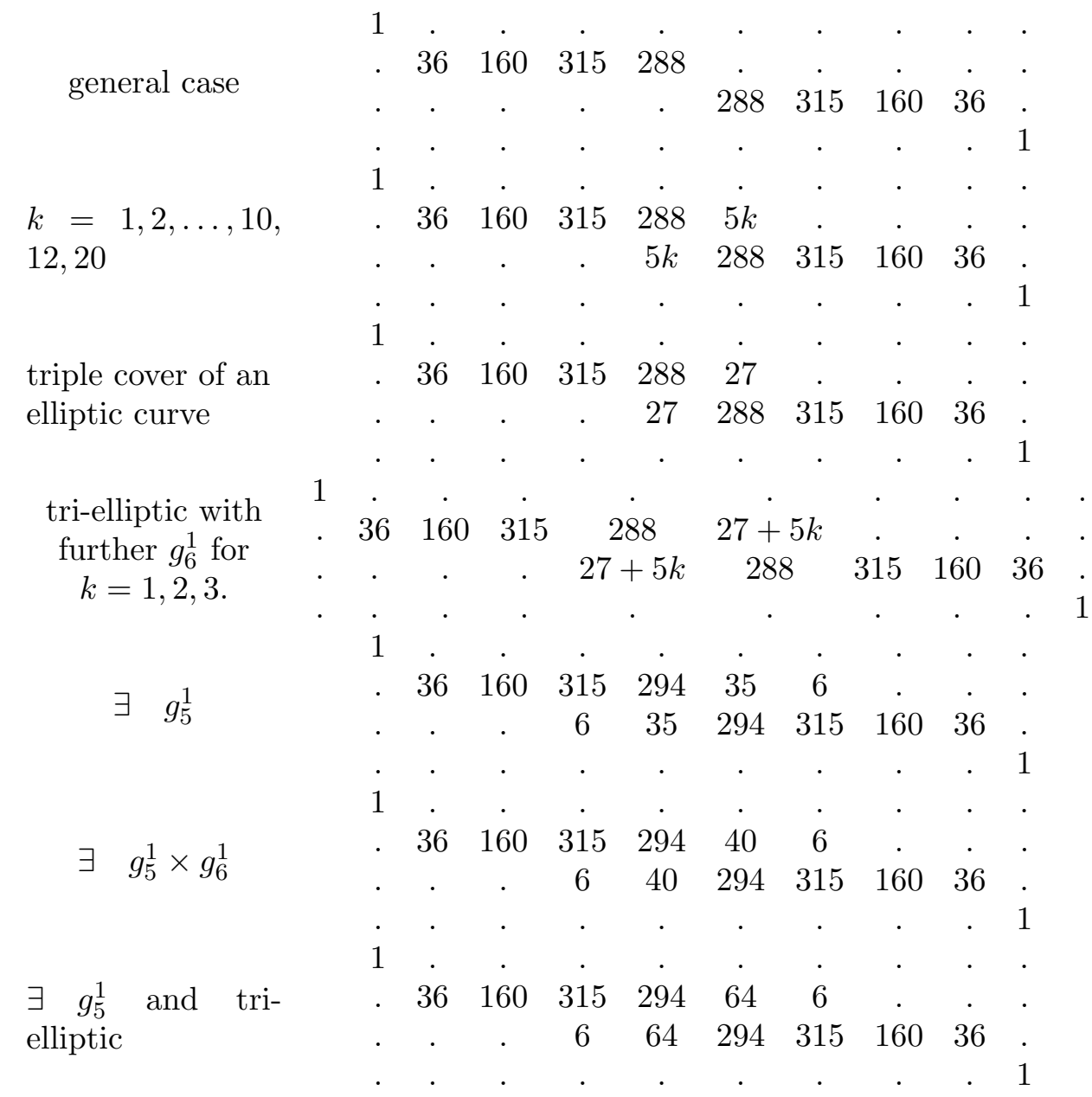




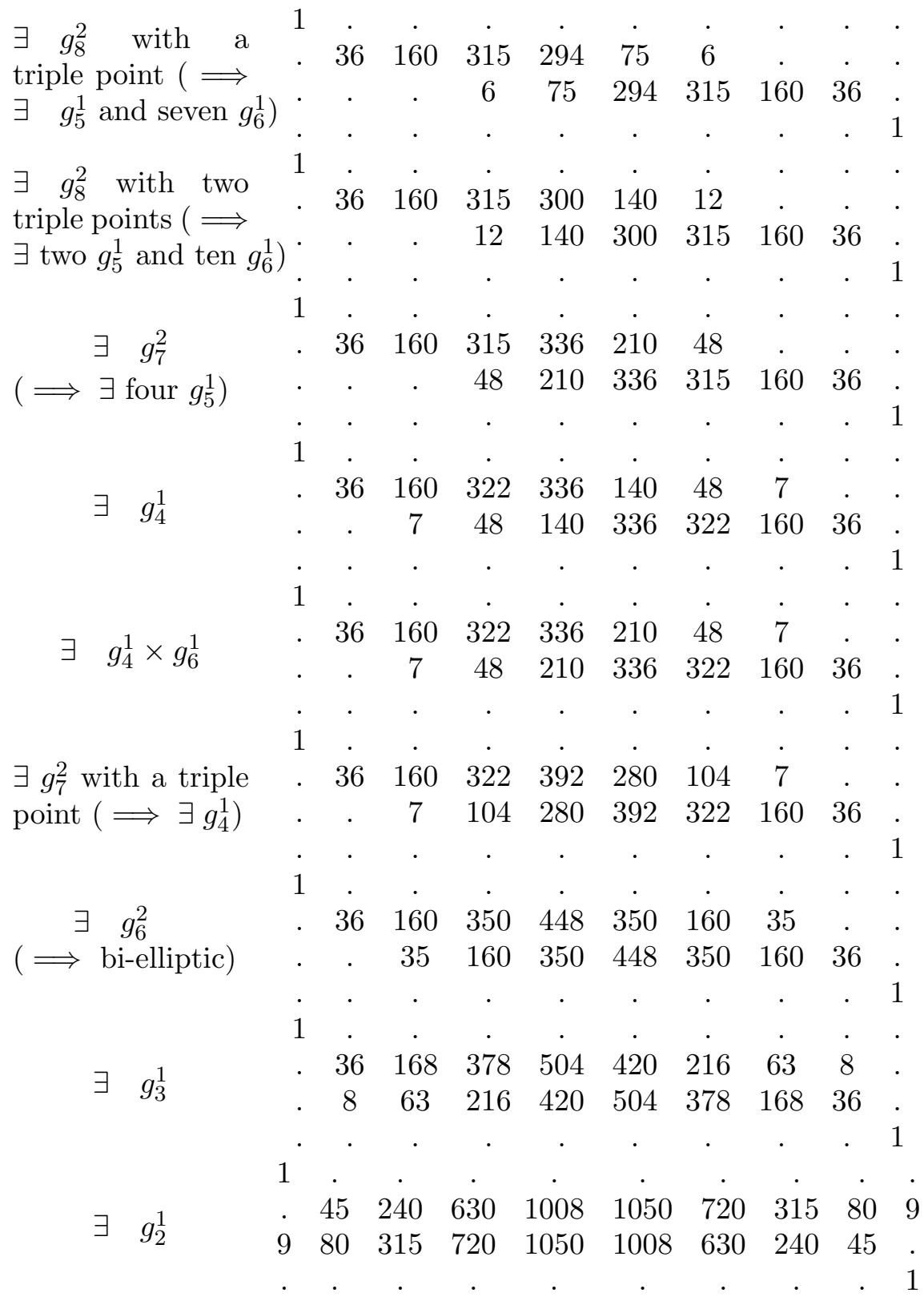

Table: Conjectural Betti numbers for genus 11, characteristic $\neq 2,3$

Some remarks are in place: The case $\beta_{46}=50$ occurs, when $C$ is a double cover of a plane quartic, or, if $C$ has a birational $g_{8}^{2}$ with nodes in general position. A special position of the nodes might result in an extra $g_{6}^{1}$ and $\beta_{46}=60$. A curve which is simultaneously tri-elliptic and a double cover of 
a plane quartic has $\beta_{46}=100$. The same number occurs for curves with a $g_{10}^{3}$. I am not certain whether the number $k$ in these tables can always be interpreted as the number of extra $g_{6}^{1}$ 's counted suitably.

I do not present a conjectural table of possible Betti numbers for higher genera. Conjecturally exceptional characteristics are summarized in the following table:

\begin{tabular}{ccc} 
genus $g$ & characteritic $p$ & extra syzygies \\
\hline$\leq 6$ & none & -- \\
7 & 2 & $\beta_{24}=1$ \\
8 & none & -- \\
9 & 3 & $\beta_{35}=6$ \\
10 & 3 & $\beta_{35}=1$ \\
11 & 2,3 & $\beta_{46}=28,10$ \\
12 & 2 & $\beta_{46}=1$ \\
13 & 2,5 & $\beta_{57}=? ?, 120$
\end{tabular}

Table: Exceptional characteristics and Betti numbers of a general curve

What is the evidence for the correctness of these tables? First the exceptional characteristics in the case of the even genera are really counter examples to Green's conjecture, because in these cases the number of additional syzygies is too small to come from a linear system. However that the generic curve of that genus has extra syzygies is not fully established. We just have a probabilistic argument, as in the case of the odd genera. For $g=9$ we can use Mukai's theorem (1995), which says that all curves of Clifford index 4 are transversal sections $C=X \cap \mathbb{P}^{8}$ of the symplectic Grassmanian

$$
X=L G(3,6) \subset \mathbb{P}^{13}
$$

of Lagrangian subspaces, and compute the syzygies of $X$ for various small $p$. The evidence is then based on our believe that exceptions occur only for small $p$. For larger odd genera we can compute examples for each small $p$. Our evidence is, that it is unlikely, that we always hit the loci of curves with extra syzygies, if we pick random different examples.

For the table of all possible Betti numbers we know for odd genus $g=2 k+1$ by Hirschowitz and Ramanan (1998), that curves with extra syzygies lie in the locus $M_{g}\left(g_{k+1}^{1}\right)$ of curves with a $g_{k+1}^{1}$ and that $\beta_{k-1, k+1} \geq k$ with equality on an open set of $M_{g}\left(g_{k+1}^{1}\right)$. On the other side every smooth curve of any genus with $\beta_{i, i+2} \neq 0$ for $i \leq 2$ satisfies Green's Conjecture by Max Noether (1880), Petri (1923), Voisin (1988) and Schreyer (1991), and their Betti numbers are computed in (Schreyer, 1986). So for odd genus $g \leq 11$ the Betti numbers are not yet known only in the case of Clifford index 3 and $g=9$ and Clifford index 3,4 and $g=11$. Turning to Green's Conjecture the only open question in this range is, whether a curve of genus 
$g=11$ and Clifford index 4 satisfies $\beta_{35}=0$. However there could be many more cases of possible Betti tables. To get some confidence in its completeness, we can do the following. Take a small characteristic $p$, say $p=5$, and construct curves in each stratum at random over $\mathbb{Z} / 5$. Then since $\beta_{k-2, k}=\beta_{k-1, k}$ we know that this number jumps up in codimension 1 . Hence we roughly expect to see such a phenomenon with a chance of $1: p$. If this expectation turns out to be true, and no new Betti tables are found, we may have some more confidence. In particular I expect, that $\beta_{46}=100$ is the maximum possible value for curves of genus $g=11$ and Clifford index 4. In this stratum I did not find any jumps at all. I checked this over $\mathbb{Z} / 5$ running 20 random examples observing no jumping up. The probability, that all twenty curves miss the jump loci in this stratum, is (if the jump loci is nonempty) roughly $0.8^{20}=0.0115$. So in some sense we can be certain with $98 \%$ that this jump loci is really empty.

For even genus $g=2 k \mathrm{I}$ am less confident. By Voisin's result (2001) we know, that Green's conjecture holds for the general curve and for general $q$-gonal curves. On the other hand there is no apriori reason why jumps in Betti numbers, say for $\beta_{k, k+1}=\beta_{k-2, k-1}$, occur in small codimension. The loci of curves with $\beta_{k, k+1} \neq 0$ is reducible for $g=10$. We have the loci

$$
M_{10}\left(g_{5}^{1}\right) \text { and } M_{10}\left(\text { half canonical } g_{9}^{3}\right) \text {, }
$$

where $M_{g}\left(g_{d}^{r}\right)=$ \{curves of genus g with a $g_{d}^{r}$, which have dimension 25 and 21 hence codimension 2 and 6 in $M_{10}$ respectively. None of these strata lies in the closure of the other.

\section{References}

[1] Bayer, D. and D. Mumford. What can be computed in algebraic geometry? In D. Eisenbud et al., editors, Computational algebraic geometry and commutative algebra. Proceedings of a conference held at Cortona, Italy, June 17-21, 1991, pages 1-48. Cambridge University Press, Cambridge, 1993

[2] Bayer, D. and M. Stillman. A theorem on refining division orders by the reverse lexicographic order. Duke Math. J., 55:321-328, 1987.

[3] Beilinson, A. Coherent sheaves on $\mathbb{P}^{n}$ and problems of linear algebra. Functional Anal. Appl., 12:215-216, 1978.

[4] Bernšteĭn, I. N., I. M. Gel'fand, and S. I. Gel'fand. Algebraic vector bundles on $\mathbb{P}^{n}$ and problems of linear algebra. Functional Anal. Appl., 12:212-214, 1978.

[5] Bodnar,G. and J. Schicho. Automated resolution of singularities for hypersurfaces. J. Sym. Comp., 30:401-428, 2000.

[6] Brieskorn, E. Die Monodromie der isolierten Singularitäten von Hyperflächen. Manuscr. Math., 2:103-161, 1970.

[7] Buchberger, B. Ein Algorithmus zum Auffinden der Basiselemente des Restklassenrings nach einem nulldimensionalen Polynomideal. $\mathrm{PhD}$ thesis, Leopold-FranzensUniversität, Innsbruck, Austria, 1965

[8] Buchberger, B. Ein algorithmisches Kriterium für die Lösbarkeit eines algebraischen Gleichungssystems. Aequationes mathematicae, 4:374-383, 1970 
[9] Chang, M.-C. and Z. Ran. Unirationality of the moduli spaces of curves of genus 11, 13 (and 12). Invent. Math., 76:41-54, 1984

[10] Cröni, H. Invarianten binärer Formen. Dissertation, Saarbrücken, 2002.

[11] Decker, W., L. Ein, and F.-O. Schreyer. Construction of surfaces in $\mathbb{P}^{4}$. J. Algebraic Geom., 2:85-237, 1993

[12] Decker, W. and D. Eisenbud. Sheaf algorithms using the exterior algebra, in D. Eisenbud et al., editors, Mathematical computations with Macaulay2. Springer, New York, 2001

[13] Decker, W., G.-M. Greuel, and G. Pfister. Primary decomposition: algorithms and comparisons, In B.H. Matzat et alt., editors, Algorithmic algebra and number theory, Heidelberg 1997, pages 187-220. Springer, Berlin, 1999

[14] Decker, W., A. E. Heydtmann, and F.-O. Schreyer. Generating a noetherian normalization of the invariant ring of a finite group. J. Symb. Comput., 25:727-731, 1998

[15] Decker, W. and T. de Jong. Gröbner bases and invariant theory. In B. Buchberger and F. Winkler, editors, Gröbner bases and applications, Linz 1999, pages 61-89. Cambridge University Press, Cambridge, 1999

[16] Decker, W., T. de Jong, G.-M. Greuel, and G. Pfister. The normalization: a new algorithm, implementation and comparisons. In P. Draexler et al., editors, Computational methods for representations of groups and algebras. Proceedings of the Euroconference in Essen, Germany, April 1-5, 1997, pages 267-285. Birkhäuser, Basel, 1999

[17] Decker, W. and S. Popescu. On surfaces in $\mathbb{P}^{4}$ and 3 -folds in $\mathbb{P}^{5}$. In N. J. Hitchin et alt, editors, Vector bundles in algebraic geometry, Durham 1993, pages 69-100. Cambridge University Press, Cambridge, 1993

[18] Decker, W. and F.-O. Schreyer. Non-general type surfaces in $\mathbb{P}^{4}$ : Some remarks on bounds and constructions. J. Symb. Comput., 29:545-582, 2000

[19] Decker, W. and F.-O. Schreyer. Computational algebraic geometry today. In C. Ciliberto et al. (eds): Application of Algebraic Geometry to Coding Theory, Physics and Computation, 65-119, Kluwer, 2001

[20] Derksen, H. Computation of invariants for reductive groups. Adv. Math., 141:366384, 1999

[21] Dixmier, J and D. Lazard. Minimal number of fundamental invariants for binary forms of degree 7. Port. Math., 43:377-392, 1986.

[22] Eisenbud, D. Green's conjecture: An orientation for algebraists. In D. Eisenbud and C. Huneke, editors, Free resolutions in commutative algebra and algebraic geometry, Proc. Conf., Sundance/UT (USA) 1990. Jones and Bartlett Publishers, Boston 1990

[23] Eisenbud, D. Open problems in computational algebraic geometry. In D. Eisenbud, editor, Proceedings of a conference held at Cortona, Italy, June 17-21, 1991, pages 49-70. Cambridge University Press, Cambridge, 1993

[24] Eisenbud, D. Computing cohomology. A chapter in W. Vasconcelos, Computational methods in commutative algebra and algebraic geometry. Springer, Berlin, 1998

[25] Eisenbud, D., G. Fløystad, and F.-O. Schreyer. Sheaf cohomology and free resolutions over the exterior algebra. Preprint, http://arXiv.org/abs/math.AG/0104203, 2001.

[26] Eisenbud, D., C. Huneke, and W. Vasconcelos. Direct methods for primary decomposition. Invent. Math., 110:207-235, 1992

[27] Eisenbud, D. and F.-O. Schreyer. Chow forms via exterior syzygies. To appear, preprint 2001. 
[28] Ellingsrud, G. and Ch. Peskine. Sur les surfaces lisse de $\mathbb{P}^{4}$. Invent. Math., 95:1-12, 1995.

[29] Encinas, S and O. Villarmajor. Good points and constructive resolution of singularities. Acta Math., 181:109-158, 1998.

[30] Gianni, P. and B. Trager. Integral closure of Noetherian rings. In W. W. Kuechlin, editor, Proceedings of the 1997 international symposium on symbolic and algebraic computation, ISSAC '97, Maui, HI, USA, July 21-23, 1997. ACM Press, New York, 1997

[31] Gianni, P., B. Trager, and G. Zacharias. Gröbner bases and primary decomposition of polynomial ideals. J. Symb. Comput., 6:149-167, 1988

[32] Gerard, R. and A. Levelt. Invariant mesurant l'irrégularité en un point singulier des systèmes d'équations différentielles linéares. Ann. Inst. Fourier, 23:157-195, 1973.

[33] Gordan, P. Neuer Beweis des Hilbertschen Satzes über homogene Funktionen, Nachrichten König. Ges. der Wiss. zu Gött., 240-242, 1899

[34] Grauert, H. Über die Deformation isolierter Singularitäten analytischer Mengen. Invent. Math., 15:171-198, 1972

[35] Green, M. Koszul cohomology and the geometry of projective varieties. J. Differ. Geom., 19:125-167, 1984

[36] Green, M. and R. Lazarsfeld. The nonvanishing of certain Koszul cohomology groups. Appendix to (Green, 1984), 1984

[37] Green, M. and R. Lazarsfeld. On the projective normality of complete linear series on an algebraic curve. Invent. Math., 83:73-90, 1986

[38] Green, M. and R. Lazarsfeld. Some results on the syzygies of finite sets and algebraic curves. Compositio Math., 67:301-314, 1988

[39] Greuel, G.-M. Computer algebra and algebraic geometry-achievements and perspectives. J. Symb. Comput., 30:253-289, 2000

[40] Greuel, G.-M. and G. Pfister. Advances and improvements in the theory of standard bases and syzygies. Arch. Math., 66:163-176, 1996

[41] Hermann, G. Die Frage der endlich vielen Schritte in der Theorie der Polynomideale. Math. Ann., 95:736-788, 1926

[42] Hilbert, D. Über die vollen Invariantensysteme. Math. Ann., 36:473-534, 1890

[43] Hilbert, D. Über die Theorie der algebraischen Formen. Math. Ann., 42:313-373, 1893

[44] Hironaka, H. Resolution of singularities of an algebraic variety over a field of characteristic zero. I, II. Ann. Math., II. Ser., 79:109-203, 205-326, 1964

[45] Hirschowitz, A. and S. Ramanan. New evidence for Green's conjecture on syzygies of canonical curves. Ann. Sci. Ec. Norm. Super., IV. Ser., 31:145-152, 1998

[46] Hochster, M. and J. Roberts. Rings of Invariants of Reductive Groups Acting on Regular Rings are Cohen-Macaulay. Adv. in Math., 13:115-175, 1974

[47] Horrocks, G. and D. Mumford. A rank 2 vector bundle on $\mathrm{P}^{4}$ with 15,000 symmetries. Topology, 12:63-81, 1973.

[48] de Jong, T. An algorithm for computing the integral closure. J. Symb. Comput., 26:273-277, 1998

[49] Kaltofen, E. Polynomial factorization. In B. Buchberger et al., editors, Computer algebra, pages 95-113. Springer, Wien, 1982

[50] Kaltofen, E. Polynomial factorization 1987-1991. In D.V. Chudnovsky and R.D. Jenks, editors, Proceedings of LATIN'92, Sao Paulo, pages 294-313. Springer, New York, 1992

[51] Kemper, G. Calculating invariant rings of finite groups over arbitrary fields. J. Symb. Comput., 21:351-366, 1996 
[52] Kemper, G. An algorithm to calculate optimal homogeneous systems of parameters. J. Symb. Comput., 27:171-184, 1999

[53] Kemper, G. and A. Steel. Some algorithms in invariant theory of finite groups. In P. Draexler et al., editors, Computational methods for representations of groups and algebras. Proceedings of the Euroconference in Essen, Germany, April 1-5, 1997, pages 267-285. Birkhäuser, Basel, 1999

[54] Kollár, J. Sharp effective Nullstellensatz. J. Am. Math. Soc., 1:963-975, 1988

[55] Kollár, J. Effective Nullstellensatz for arbitrary ideals. J. Eur. Math. Soc., 1:313337, 1999

[56] Macaulay, F. Some properties of enumeration in the theory of modular systems. Proc. London Math. Soc., 26:531-555, 1927

[57] Martin, B. Computing versal deformations with SINGULAR. In B.H. Matzat et alt., editors, Algorithmic algebra and number theory, Heidelberg 1997, pages 283-293. Springer, Berlin, 1999

[58] Mayr, E. W. Some complexity results for polynomial ideals. J. of Complexity, 13:305-325, 1997

[59] Mayr, E. W and A. R. Meyer. The complexity of the word problems for commutative semigroups and polynomial ideals. Adv. Math., 46:305-329, 1982

[60] Mora, T. An algorithm to compute the equations of tangent cones. In J. Calmet, editor, Computer algebra, EUROCAM '82, Conf. Marseille/France 1982, pages 158-165. Springer, Lecture Notes in Computer Science 144, Berlin, 1982

[61] Mori, S. and S. Mukai. The uniruledness of the moduli space of curves of genus 11. In M. Raynaud and T. Shioda, editors, Algebraic geometry, Proc. Jap.-Fr. Conf., Tokyo and Kyoto 1982, pages 334-353. Springer, Lecture Notes in Mathematics 1016, Berlin, 1983

[62] Mukai, S. Curves and symmetric spaces. I. Am. J. Math., 117:1627-1644, 1995

[63] Noether, E. Der Endlichkeitssatz der Invarianten endlicher Gruppen. Math. Ann., $77: 89-92,1916$

[64] Noether, E. Der Endlichkeitssatz der Invarianten endlicher linearer Gruppen der Charakteristik p. Nachr. v. d. Ges. d. Wiss. zu Göttingen, 28-35, 1926

[65] Noether, M. Über die invariante Darstellung algebraischer Funktionen. Math. Ann., 17:263-284, 1880

[66] Petri, K. Über die invariante Darstellung algebraischer Funktionen einer Veränderlichen. Math. Ann., 88:242-289, 1923

[67] Richman, D. R. On vector invariants of finite fields. Adv. in Math., 81:30-65, 1990

[68] Gröbner deformations of hypergeometric differential equations. Springer, Berlin, 2000

[69] Schreyer, F.-O. Syzygies of canonical curves and special linear series. Math. Ann., 275: 105-137, 1986

[70] Schreyer, F.-O. A standard basis approach to syzygies of canonical curves. J. Reine Angew. Math., 421: 83-123, 1991

[71] Schreyer, F.-O. Small fields in constructive algebraic geometry. In M. Maruyama, editor, Moduli of vector bundles, pages 221-228. Marcel Dekker, New York, 1996

[72] Schreyer, F.-O. and F. Tonoli. Needles in a haystack: Special varieties via small fields. In D. Eisenbud et al., editors, Mathematical computations with Macaulay2. Springer, New York, 2001

[73] Seidenberg, A. Construction of the integral closure of a finite integral domain. II. Proc. Am. Math. Soc., 52:368-372, 1975

[74] Sernesi, E. L'unirazionalita della varieta dei moduli delle curve di genere dodici. Ann. Sc. Norm. Super. Pisa, Cl. Sci., IV, 8:405-439, 1981 
[75] Shimoyama, T. and K. Yokoyama. Localization and primary decomposition of polynomial ideals. J. Symb. Comput., 22:247-277, 1996

[76] Smith, G. G. Computing global extension modules. J. Symb. Comput., 29:729-746, 2000

[77] Stanley, R. B. Invariants of Finite Groups and their Applications to Combinatorics. Bull. Am. Math. Soc., 1:475-511, 1979

[78] Sturmfels, B. Algorithms in Invariant Theory. Springer, Wien, 1993

[79] Traverso, C. Hilbert functions and the Buchberger algorithm. J. Symb. Comput., 22:355-376, 1996

[80] Vasconcelos, W. V. Computing the integral closure of an affine domain. Proc. Am. Math. Soc. , 113:633-638, 1991

[81] Voisin, C. Courbes tétragonales et cohomologie de Koszul. J. Reine Angew. Math., 387:111-121, 1988

[82] Voisin, C. Green's generic syzygy conjecture for curves of even genus lying on a K3 surface. Preprint, Paris, France, 2001

[83] Wang, D. Characteristic sets and zero structures of polynomial sets. Preprint RISCLINZ, Linz, Austria, 1989

[84] Walter, U. Cohomology with rational coefficients of complex varieties. Preprint, 2000

[85] Walter, U. D-modules and the cohomology of varieties. In D. Eisenbud et al., editors, Mathematical computations with Macaulay2. Springer, New York, 2001

[86] Wester, M. (ed.) Computer algebra systems. A practical guide. Wiley, Chichester, 1999

[87] Winkler, F. Polynomial algorithms in computer algebra. Springer, Wien, 1996

F-O SChreyer: Universität DES SAARLANDES,

FAKUltät Für Mathematik Und Informatik, Geb. 26,

D-66123 SaArbrǘcken, Germany.

E-mail address: schreyer@math.uni-sb.de 